\title{
Chiptune: The Ludomusical Shaping of Identity
}

\section{George Reid ${ }^{1}$}

Received: 28 September 2018 / Accepted: 3 October 2018 / Published online: 20 October 2018

(c) The Author(s) 2018

\begin{abstract}
The creativity of chiptune fandom is as expansive as it is stylistically recognisable; composers may venture into the stylistic tropes of early videogame music, jazz, or a rendition of the theme for Miami Vice, yet they consistently retain a distinct and stylistically 'limited' microsound character. Distinctly chiptune in timbre, but stylistically or thematically reaching beyond chiptune's videogaming origins, how do these creative and (sub)cultural intersections relate to chiptune fan identity and furthermore, what do they reveal about the broader implications of the relationship between music and identity? This article presents an interdisciplinary framework for analysing the relationship between the musical creativity of chiptune fandom and the identities of its composers, constructed through theoretical influences from ludomusicology, fan studies, and Rosi Braidotti's critical theory of 'nomadic subjectivity.' The research intention of this article—as part of a larger project—is to develop an analytical framework that not only applies to the relationship between chiptune creativity and fan identity, but also forms an adaptable lens for all music and media creativity undertaken in view of fannish identification.
\end{abstract}

Keywords Chiptune $\cdot$ Fandom $\cdot$ Identity $\cdot$ Ludomusicology $\cdot$ Play $\cdot$ Videogames

\section{Introduction}

Chiptune — or as it is sometimes known 'chipmusic' or 'micromusic'—describes the microsound genesis of early videogame sound and music. The contemporary creativity of its now global — and 'glocal'-fandom, is broad, heterogeneous and, as this paper argues, ever expanding at the ludomusical hands of its equally diverse participants (see Wellman 2004 on glocality, pp. 26-29). Any single creative chiptune fan

\footnotetext{
All editorial decisions for this article, including selection of reviewers and the final decision, were made by guest editor Dr. Kenny McAlpline.
}

George Reid

k1455076@kingston.ac.uk

1 Kingston University, Kingston Upon Thames, London, UK 
can compose an expansive range of music, which may adhere to the stylistic conventions of videogame soundtracks of the 1980s and 1990s, or branch into other genre conventions, tropes, and media franchises. We can observe, for example, chiptune (sub)genres of bitpop, 8-bit Reggae and many other mixtures between chipsound and more contemporary-or even other retro-styles; 'authentic' early Nintendo and Game Boy style chiptunes on original microchip hardware; 'fakebit' as larger scale chiptune arrangements utilising modern audio production equipment and virtual emulations of microsound hardware; and overtly intertextual 8-bit renditions of Phil Collins and 16-bit Super Nintendo imaginings of the theme to the BBC scifi television series Doctor Who (1963-1989, 2005). Chiptune continues to spread into innumerable musical and media genres, niches, and intertextuality, and is as stylistically and thematically broad as it is semi-consistent in its microsound or 'bitcrushed' timbres.

Given this creative expanse, and furthermore the demographic heterogeneity of chiptune fans, how can we understand chiptune creativity in relation to the identity of the composer, especially if her creativity is explicitly intertextual? Furthermore, how does chiptune intertextuality appeal to its fans, and likewise simultaneously appeal to multiple points of fannish identification in a single musical artifact? Is chiptune fan identity, therefore, equally as multifaceted and complex as its creativity? If so, how can we account for this fluidity and what does it reveal about the construction of fan identity through musical interaction?

\section{Theoretical Background}

While there are academic works meticulously dedicated to the history, technology, and compositional heritage of chiptune fandom, chiptune fan identity has yet to receive academic analysis to the same extent as other creative music, media, or literary fandoms. This said, there are works beginning to address chiptune creativity in both its creative expanse and (sub)cultural footprints. Nicolas Nova's text 8-bit Reggae: Collision and Creolisation for example; though not intended as academic, Nova does provide a fascinating case study. Jamaican 'riddims' realised through the Commodore 64's SID chip reveals a dynamic play of (sub)cultural genealogies and identities, which Nova does trace through interviews and textual analyses (Nova 2014, passim). While the presented interviews reveal both reggae and chiptune to be distinctly important to the composer's sense of identity, the scope of Nova's investigation does not provide an analysis for how exactly the combination of reggae and chiptune produces this affirmation.

Mike D'Errico's 2011 paper How to Reformat the Planet: Technostalgia and the 'Live' Performance of Chipmusic is another noteworthy text on chiptune practice. Discussing chiptune's 'technostalgia' with insight from Jacques Derrida's notion of 'hauntology,' D'Errico's work makes the important suggestion that chiptune's nostalgic effect is rather a result of its obsolete compositional practices and distinctly 'retro' sound (2012). This notion suggests that the interfaces and sonic qualities of chiptune's microchip technologies impact identity through their agency-one that is non-human. Both Nova and D'Errico's works are important 
in revealing the complexity of chiptune fan identity, pointing towards why the approach to chiptune analysis must consider its creative breadth and must furthermore theorise how chiptune creativity perpetuates a sense of self and fandom. Both research areas are charged with potential, and yet currently remains theoretically underdeveloped in (ludo)musicological studies dedicated to chiptune. As such, providing an insight into this relationship is the intention of this article, which demonstrates the theoretical framework from my larger $\mathrm{PhD}$ project on chiptune, fan identity, and its perpetuation through ludomusicality.

In the $\mathrm{PhD}$ project, the on-going process between chiptune and fan identity is understood through a (ludo)musicological adaptation of Rosi Braidotti's theory of nomadic subjectivity: a critical theory that in part dedicates focus to the trans/ formation of identity through human and non-human relationality (2011a, b). Adapted for (ludo)musical use, Braidottian nomadism forms both the theoretical background and navigational framework for analysing the chip-musical 'shaping' of chiptune fan identity through its fannish consumption (cf. Sandvoss 2005, pp. 154, 157). Through Braidotti's influence, the theoretical framework necessarily focusses on both human and non-human agency in the process of chiptune and fan identity, therefore avoiding the essentialist trappings of strictly anthropocentric or technologically deterministic approaches (cf. Cheng 2014, p. 8; see also Braidotti 2011a, 1. 2398).

Analysed as 'nomadic' in the Braidottian sense, the main argument of the thesis-and thus this article-contends that the 'performance' and affirmation of chiptune fan identity is continuously reliant on nomadic, affective encounters between fannish desire and the agency of non-human, chip-musical 'encounters.' In turn, creative chiptune fans have the agency to engender these encounters in view of fannish identification through their ludomusicality. This process can be split into three areas of argument and analysis:

1. Chiptune's fannish identifications are contingent on chip-musical immersion, in which listeners nomadically encounter the affective, non-human agencies of chipsound 'actors.' This agency triggers webs of mnemonic and fannish associations

2. The creative agency of the chiptune composer in 'tailoring' the chip-musical encounters-suited to her sense of identity and fandom - through her chosen chipsound practice and aesthetic, and thus chiptune composition operates as a nomadic practice — and process - of identification and affirmation

3. However, while human agency plays a distinct role in the process of chiptune's fannish identification, the actualisation of fandom is always reliant-in tandemwith non-human agency. Thus, while the creative chiptune fan has agency in influencing chip-musical encounters through composition, the process of fannish identification is equally reliant on the non-human agency of her compositional tools and their sonic mediating capacities. This reveals the entire process of chiptune fandom to be nomadically relational between human and non-human agencies 
This article demonstrates the theoretical framework of these lenses through the analysis of an 'intertextual' chiptune, one that rearranges and remixes popular or other film/television/videogame music through chiptune's emblematic timbres, stylistic tropes and compositional practices (cf. D'Errico 2012).The specific focus on chiptune intertextuality and its nomadic relationship to the formation of fan identity intends to open the door for understanding the more nuanced affirmations of chiptune fan identity through music. In addition, beyond the scope of chip-musical creativity, further insight into how choices in musical creativityas both thematic and aesthetic-directly influence the trans/formation of identity, including how differing compositional choices can engender differing notions of fandom and self.

\section{Section 1:'Starman 64'-Chiptune's Creative Expanse}

Let us take the case study of a Commodore 64 SID arrangement of David Bowie's Starman, coded in 2016 as a posthumous tribute by chip-musician 'Rock' for a larger demoscene project by 'Elysium' and 'Finnish Gold.' We recognise the tune as Starman, but its arrangement, its character and microchip 'sound' now reach out and into a distinctly different (sub)cultural context.

Bowie's melody is now voiced through a single sawtooth wave, which follows the lyrical and vocal intonations of the original as it modulates through the characteristically nasal filter of the SID chip. In a single audio channel, the harmony is conveyed through the rapid arpeggio technique-a common practice in chiptune and, incidentally, now a stylistic trope. Mick 'Woody' Woodmansey's drums are now sounded through trills of filtered white noise, and the stuttering, almost Morse Code-like octaves on electric piano and guitar in the pre-chorus find their new home in 8-bit bleeps. Bowie's Starman has been recontextualised and transformed through the mediation of the Commodore 64's SID chip to become a chiptune.

As 8-bit Starman demonstrates par excellence, chiptune creativity is not hermetically bound to the rubric of videogaming culture. As discussed in the introduction, the academic fascination here is the relationship between this musical intersection of Bowie's interstellar nursery rhyme, the timbral qualities and idiosyncrasies of the Commodore 64, and the identity of the composer. How does such creativity relate to chiptune fan identity and how can we theorise this relationship given that chiptune creativity is so multifaceted, multilayered, often expansively intertextual and complex? How can we understand such a musical piece as relating to chiptune identity if it includes an explicit connection to David Bowie?

\section{8-Bit Bowie as Ludomusical Fannish Creativity}

Such intertextual creativity as 8-bit Starman is typical of a fan identity: an identity or persona created in commitment for a media text or object of personal and emotional significance (Sandvoss 2005, pp. 1-5 passim; see also Duffett 2013).Though 
this field of research is largely in relation to media and literary fans, its theories can also be applied to such intertextual chiptune creativity as 8-bit Starman.

Fandom itself, as Matt Hills states, is 'fluid, multiple, and dynamic [...]' (2014, pp. 18-19). Through their creativity, fans can simultaneously enact many different personal fandoms as a form of intertextual citation (cf. Hills 2002). These citations allow for fans to create new material 'in the margins' of existing texts by 'poaching' elements of existing media-fan fiction, for example, combines literary elements of multiple media texts as a resource to create new texts (Jenkins 2013, p. 353).

Adapting this notion of fannish intertextuality in a musicological context, such creativity can be understood as formed through 'ludomusical' processes. Roger Mosley defines ludomusicality as playful musical processes and gestures, but not strictly in relation to instrumentality (2016, pp. 15-17). Ludomusicality can indeed extend to playing in, with, and around the limitations of a medium, or pre-existing musical and (sub)cultural contexts (cf. Ibid, p. 42). We can apply this concept to intertextual chiptune creativity; 8-bit Starman sees an exercise in creative freedom around and beyond the limitations of micro-sound chip technology, and also a very fannish intertextual citation of multiple points of fannish identification through the citation of Bowie. Through its conflation of these (sub)cultural citations, 'Rock's' piece also ludomusically plays with and mediates the chronology of temporality and memory (Van Dijck 2007, pp. 1, 27 \& Passim; Van Elferen 2012, p. 30).

As a synchronous mixture of music fandoms, 8-bit Starman demonstrates the fannish intertextuality of chiptune's ludomusical creativity and, thus, the potential complexity and multi-faceted repertoires of fannish attachments present in the makeup of 'Rock's' chiptune fan identity. The analytical challenge presented by 8-bit Starman is to understand how the intertextual creativity of chiptune audio culture fluidly produces and 'shapes' fan identity, without resorting to inadequate and structural identity models pertaining to musical essences and 'authenticities.' As relationships between music and identity are always in flux, changing, comprised of multiple memories and levels of experience, we can here gain insight from Rosi Braidotti's theory of 'nomadic subjectivity.'

\section{Section 2: Chiptune-Nomadic Theorisation}

Braidotti is a feminist philosopher, whose theoretical framework of nomadic subjectivity focuses on identities and subjectivities in on-going and non-linear flux. The nomadic subject's sense of 'self' and identity is continuously synthesized in relation to affective encounters with other entities (2011a, 1. 422-343, and Passim; cf. Massumi 2002, 1. 601, 1089). This is not to say that subjectivity is entirely at the 'mercy' of external agencies; Braidotti also understands desire as sustaining subject formation, and the meeting of these agencies define the 'affective encounter.' She claims: 'a sense of identity [...] rests not on fixity, but on contingency' and '[...] what sustains the entire process of becoming subject is the will to know [and] the desire to speak [...]' (2011a, 1. 422-447). Meaning, subjectivity is a relational flow between inner desire and external or outer affectivity, and that the nomadic subject can willingly enact a desired synthesis of the 'self' through encounters it deems as 
contingent to its identity. This synthesis of the self is only temporary, however, as the nomadic subject is never 'fixed' by these syntheses of encounters and desires. How, then, can we relate Braidotti's notion of nomadic syntheses of identity to intertextual chiptune creativity and, furthermore, chiptune identity?

In a similar process to Braidotti's nomadic subjectivity, the temporary construction of chiptune listening subjectivity is reliant on an affective encounter-chiptunes and the sonic qualities of microsounds in this case-and thus it can be proposed that chiptune composers can 'tailor' or 'impact' the encounter and the form that subjectivity may take. So, while chip-musical encounters may be essential for the chiptune fan to feel 'who' they are or to which (sub)culture they belong, chiptune composers are capable of tailoring this sensation through compositional choices. I argue that this process takes place through the interaction of two main vectors: the first is the nomadic construction of listening subjectivity through the affective agency of chiptune encounters. The second vector of fannish desire is the influence of the chiptune artist's self-definition - as how they wish to produce and enact their sense of identity-on their compositional practice.

Three important questions can help demonstrate what I mean here: First, how does chiptune listening subjectivity operate in relation to chiptune fan identity? Second, how is chiptune contingent to the nomadic 'form' of subjectivity? And finally, how do chiptune composers influence the construction of their listening subjectivity in relation to their sense of identity? Answering these questions require first theorising chiptune listening subjectivity and how it is formed nomadically through chipmusical networks. Once this is established, the analysis can then focus on how these chip-musical networks, in turn, are influenced by the chiptune fan in compositional choices based on subjective notions of her (sub)cultural identity narratives.

\subsection{Chiptune, Listening Subjectivity and Fan Identity}

Subjectivity, in Braidotti's understanding, is an ever-changing amalgam of heterogeneous - but relationally interconnected-levels of experience with other entities and personal desires (2011a, 1. 422-34; 2013, p. 8). It is therefore important to establish that at any given time, subjectivity does not operate at a single level of experience or memory but is instead constructed as an interconnected amalgam of different experiences, memories and emotional connections. Furthermore, subjectivity is comprised of 'posthuman' elements, meaning that they can consist of experiences with human and non-human entities (Braidotti 2013, pp. 3-8). Being nomadic, these amalgams of subjectivity are continuously shifting in their collective makeup.

For the context of chiptune fan subjectivity, the amalgam of 'entities' referred to here are musical/non-musical, human/non-human 'actors.' This notion has been theorised by Isabella van Elferen and Charlie Blake in their application of Latourian actor network theory (ANT) to musical listening (2015; 2005). Expanding ANT to incorporate musicology, they contend that musical and non-musical actors such as melody, rhythm, timbre, harmony, articulation, and technological mediation all converge in musical encounters, and all have an impact upon the listener's subjectivity (2015, pp. 60-70). The subjectivity of the human listener-as an actor in the 
network-is demarked by an encounter with all actors in a musical network, not only musical ones.

For chiptune, we can understand that these actors may include non-human and non-musical 8-bit or 16-bit timbral qualities. Chiptune technology becomes a nonhuman and non-musical actor in the network; hearing, for example, that a chiptune is composed using a Sega Master System chip also contributes to listening subjectivity formation as an encounter. The listener would encounter the sound-mediating agency of the Master System chip as a non-human actor in the network, allowing for the listener to not only characterise or categorise the chiptune, but also construct subjectivity based upon how they personally relate to this encounter.

It is furthermore necessary to involve non-musical, human and non-human actors as part of subjectivity formation to understand how subjective musical meaning making arises. These can include memories, nostalgia, self-identification, videogaming pleasures and (sub)cultural 'capital' - all of these elements are triggered and, moreover, become interconnected through chiptune interaction (cf. Thornton 1995). Any convergence of these heterogeneous actors, musically and culturally intertextual, all contribute to the ephemeral construction of subjectivity, as the encounter of these musical networks is affective, and the listener relates to the encounter through memory and identification-constructing a subject position through the agency of these relations.

Latourian actor network theory is strikingly akin to Braidotti's nomadic subjectivity in the understanding that subject demarcation occurs in its relational interaction with other entities_-including posthuman elements (Latour 2005, p. 45; Braidotti 2011a, 1. 432-444). While neither of these theories involves music, they can be applied to the construction of subjectivity in the encounter with musical and non-musical actors; we attribute meaning to them based upon how they relate to our sense of 'self,' or our own memories and identity narratives. We may approach media from a sense of 'who we are,' but we are also 'changed' by the encounter (cf. Grossberg 1988 cited in Wolff 1995, p. 119).

Music, as Simon Frith states, may be 'integral to [producing] identity' (2002, p. 110); one may experience 'I am a goth' or 'I am a punk' through music, or in the case of chiptune 'I am a chiptune fan and/or artist; I belong to chiptune audio culture and the music I listen to is me.' The sound of the Commodore 64, for example, can have a profound meaning for a chiptune fan. The timbral qualities of the SID chipas musical and non-musical/technological actors-may allow the listener to experience a shift in subjectivity that relates to their own sense of identity and fandom. The agency of these actors then simultaneously 'change' the fan as much as they 'produce' and 'root' them in a sense of identity.

Such musical interaction, which Christopher Small frames as 'musicking,' sets up relationships of meaning, and such relationships may include fannish identity narratives (1998, pp. 3-6, passim). I extend this notion with the fannish desire 'to musick,' proposed by Small as a verb (1998, p. 3); as musical meaning is always a social process between musical actor networks and the knowledge of one's fandom, then to musick is to engage in this posthuman affectivity with the deliberate view to engage in their appropriated meanings and evocations of the 'self' (Blake and Van Elferen 2015, p. 65-70; Slobin cited in Frith 2002, p. 110). 
Through the music we listen to, we produce and intensify a sense of 'who' we are and 'where' we belong. However, in addition to the fluidity of fannish senses of 'self,' the sense of identity music may engender for the listener is temporary in that it is never fixed. For example, each song on an album potentially affords a different listening subjectivity - a sense of who we are in relation to that song and its attributed meanings, our subjectivities and even moods and memories consistently shifting as the CD or iPhone runs through a track list, or as we select specific tracks depending on how - or even 'who' - we feel at the time. Thus, while musical actornetworks have their own transformative agency, the listener in turn transforms them with meaning based upon memory and connotation, as filtered through their fannish and musical 'literacies' (see Van Elferen 2016, p. 32; Fritsch 2016, pp. 92-115).

Chiptune fan identity, similarly, is not a stable or singular level of experience, but instead an on-going and fluid gambol of interconnected subjectivities that arise and (re)connect through relationality between musical context, fannish appropriation and creativity. This process is not finite and does not have a static end. Chiptune fan identity has to be understood as continually synthesized, as a temporary and musically informed subjectivity, through encounters with specific chiptune aesthetics and intertextual or intercultural references in musical interaction.

The nomadism of this relationality lies in the simultaneous production of a 'located' identity or subject position — always unstable — and the susceptibility to the change in subjectivity through the on-going influence of chiptune's external, posthuman others (cf. Braidotti 2011a, 1. 181; cf. Gedalof 1999, p. 131; cf. Grossberg 1988). Chiptune participants reach outward towards posthuman relations and not solely inward in their formation of chiptune fan identity-they need the music for them to temporarily 'be' fans, be that an emotional high, nostalgia, or the enjoyment of Blip Fest. To emphasise Braidotti's notion of identity as resulting through trans/ formation, and not fixity, for chiptune fans possess a sense of who they are in relation to what they like, they need the affective encounters and transformative agency of musical actor-networks. Chiptune listening subjectivity and its identifications are therefore nomadic as not inherent, stable or permanent to the fan, but contingent on musical interaction for the listener to become-fan in relation to the chiptunes they hear.

\section{Section 3: Chiptune Composition, Fannish Desire, and the Citation of Affective Encounters}

Now that I have established the 'external' agencies that trans/form chiptune subjectivity, this analysis can now discuss the agency of the composer in engendering chipsound encounters, and the role of the 'internal,' informing factors of fannish desires. Which desires play a part in chiptune subjectivity and meaning making, and how are these all 'cited' in composition? It is not the aim of this article to define an overarching consensus of what fans may feel or desire in chiptune musicking, but I would like to argue that the appropriation of chiptune as a fannish 'Heimat' has a powerful agency in the nomadic processes of chiptune subjectivity. 
The German term 'Heimat,' translated literally as 'homeland,' is adapted by fan studies scholar Cornell Sandvoss to describe the fannish experience of media texts as a kind of 'emotional home,' through which a fan may sustain belongings and identifications (2005, pp. 64-67). But this 'home' is not entirely self-defined by human influence and does not exist outside of fannish appropriation and selfdefinition (Ibid). Fandom, as Matt Hills stresses, is not one-dimensional; fans indeed possess the knowledge of multiple Heimat locations, and these repertoires are never hermetic (cf. Hills 2014, pp. 18-19). Moreover, the time-space of the fannish Heimat is unstable as it necessarily blurs and conflates between real, physical space and the immersive space of either the textual world, or the emotional significance through which the Heimat text operates (Sandvoss 2005, pp. 66-67).

Analogously, Braidotti's notion of 'home' forms a parallel with the fannish Heimat in that the nomadic subject can have many 'homes' (2011b, 1. 603-615, 5468). Forming a connection with her notions of 'self,' the construction of these 'homes' and their afforded identifications are also not entirely self-defined, but contingent on affective and relational posthuman encounters between self and other (Ibid).

As chiptune fan identity is also dynamic and multifaceted, comprised of ever shifting webs of subjectivities, chiptune fans can similarly locate multiple senses of Heimat and fannish belonging in different chiptune aesthetics and practices. These can range from specific 8- or 16-bit sounds and practices, and their videogame console affiliations, the nuances of microsound synthesis methods, or other fannish touchstones. A part of the sustainment of chiptune fan identity, furthermore, necessarily operates through the fannish desire for these Heimat encounters and their appropriated identifications and belongings. Fannish desire as informed through a fan's self-definition, and mostly unconscious, vectors the subject towards media engagement in an active pursuit of Heimat affectivity or, as we can now consider, maintained engagement in acts of musicking (cf. Lancaster and Mikotowicz cited in Peter 2014, p. 47; Small 1998, p. 40; see also Brooker 2007). Chiptune composition can then act as the creation of a new Heimat through musically enacting desired encounters and relationality.

Chiptune composers can then be understood as being able partially to direct the chip-musical encounters they interact with, therefore influencing subject formation through musically evoking notions of Heimat. The fannish desire of the chiptune fan, as the mostly unconscious want for fannish identification, informs the processes of choosing microsound compositional aesthetics or directly intertextual references, which may engender a desirable emotional reaction or a sense of 'this is me' (cf. Brown 1996, p. 57). Chiptune composition in this light is a synthesis of influencesmusical, technological and (sub)cultural elements that fans consider to be congruent to a desired sense of 'self' and Heimat - and all these influences can become traceable within the musical actor-network. Microsound or intertextual elements are therefore 'mattering elements:' elements which fans imbue with personal investments and significance (cf. Grossberg 1992, p. 82).

The microchips chiptune fans hack or emulate, the tracker sequencers and synthesizers they feel best produce their desired 'sound,' and furthermore the evocation of other sources of fandom reveal that mattering elements are conjured in chiptune composition via means of sonic or 'musical citations,' which means that in any 
given piece multiple sources of identification can be evoked, and traced, along with their affective agencies (cf. Van Elferen 2012, p. 27; cf. Hills 2002, p. 109). This is not to suggest that these citations produce the same mattering significances for every fannish listener, especially given the subjectivity of musical meaning making, though it is important to recognise that the chiptune composer has input in deliberately interconnecting and tailoring chip-musical actor-networks as a means of constructing and overlapping subjectivities congruent to her current sense of identity and fannish belonging.

Chiptune composition in relation to fan identity is then a process of fannishly and nomadically reflecting on, and thus musically citing, 'who I (currently) am,' 'what I am a fan of' or 'where and when do I wish to evoke?' (cf. Kelsey cited in Henry et al. 2014, p. 150). But then the resulting subjectivity and subject position, as nomadic, constantly transforms and moves through subsequent affective encounters. Such encounters for chiptune fans, then, produce simultaneous processes of nomadically reflecting upon 'who' they are as fans, while also becoming 'who' they believe they are, or fannishly desire to be, if these encounters cite Heimat elements and relations. The relationship between chip-ludomusical creativity to chiptune fan identity is therefore 'nomadic' in three of the Braidottian senses:

1. Participants are not always chiptune fans, they must keep becoming chiptune fans through the posthuman agency of musical encounters.

2. Chiptune fan identity is a play of subjectivities constructed of heterogeneous musical, technological, and (sub)cultural tastes and narratives that are contingent to the participant's fannish desire and sense of 'self'

3. Chiptune composition becomes a practice of affirming and interconnecting multiple desired fannish identifications by musically citing non/musical and non/ human encounters

\section{Conclusion}

In summation of the theories proposed over the course of this article, the final question to draw everything together is: how does the framework of nomadic subjectivity relate to 8-bit Starman? For some, 8-bit Starman may be pastiche, ironic, a novelty, or as enjoyable as the original. For 'Rock' as the composer, recreating Bowie's Starman through the mediation of the Commodore 64 presents the simultaneous affirmation and performance of fandoms that, while initially unrelated in their original contexts outside of fannish creativity, both nomadically inform important parts of his identity.

Through his chiptune arrangement, 'Rock's' 8-bit Starman moves through the potential 'barriers' or striations of their pre-existing contexts involving (sub)cultural affiliations and, often, perceived 'authenticities.' The pre-existing contexts and associations of both the Commodore 64's timbral character-and microsound in a larger scope-and Bowie's original are uprooted and recontextualised, yet the citations of each fandom remain nomadically interconnected and fluid in the resulting work. 
Through this interconnection, within the time-space of 8-bit Starman the listener encounters a shifting, posthuman network of his or her own mnemonic and fannish associations in relation to the Commodore 64's 'remediation' of Bowie's work, and the haunting of Bowie's original performances (cf. Bolter and Grusin 2000, pp. 31-45).

For some fans, the agency of this piece enacts the affirmation and connection of multiple parts of an individual's identity; for others, it may be 'inauthentic.' For 'Rock', however, 8-bit Starman playfully and nomadically interconnects multiple fannish subjectivities and identifications into a single musical artifact, by citing the agencies of musical/non-musical, human/non-human actors through the informant of fannish desire. The nomadic ludomusicality of 8-bit Starman thus engenders a time-space for multiple and synchronous fannish identifications, nomadically citing and tracing existing fannish genealogies whilst, simultaneously, reproducing and interconnecting them anew.

Beyond overt intertextuality, then, we can begin to consider all of chiptune's ludomusicality as varyingly intertextual. Not only as a social performance of taste, but as the chip-musical citation of multiple fannish desires and fandoms, mattering elements, and narratives as fragments of one's own identity in the temporary construction of listening subjectivity. Just as chiptune's creativity operates through outward spiraling interconnection, so becomes its fan identity as listening subjectivity fluidly interconnects through multiple fannish Heimat identifications, just as they too are also transformed through the ludomusical manipulation of pre-existing significance and meaning.

Music fandom is not a 'destination,' but rather an ongoing, nomadic remediation and reinvention (Hills 2014, p. 20). Therefore, perpetually through the affective relationality of chiptune creativity, whichever the (sub)genre, whatever the microchip, or even whether it is composed through microchip hardware or VST - whether harking back to the golden age of videogaming, chip-tuning the Game of Thrones (HBO 2011) theme or coding 'chipped-on Bach,' chiptune fan identity is never obsolete.

Open Access This article is distributed under the terms of the Creative Commons Attribution 4.0 International License (http://creativecommons.org/licenses/by/4.0/), which permits unrestricted use, distribution, and reproduction in any medium, provided you give appropriate credit to the original author(s) and the source, provide a link to the Creative Commons license, and indicate if changes were made.

\section{References}

Blake, C., \& Van Elferen, I. (2015). Spectral media and sonic loops. In D. J. Edwards (Ed.), Technologies of the gothic in literature and culture: Technogothics (pp. 60-70). London: Routledge.

Bolter, D. J., \& Grusin, R. (2000). Remediation: Understanding new media (pp. 31-45). New York: MIT Press.

Braidotti, R. (2011a). Nomadic subjects: Embodiment and sexual difference in contemporary feminist theory (2nd edn, 1. 181-447) New York: Colombia University Press. [Kindle Edition]

Braidotti, R. (2011b). Nomadic theory: The portable Rosi Braidotti (1. 603-615, 5468). New York: Colombia University Press. [Kindle Edition].

Braidotti, R. (2013). The posthuman (pp. 3-8). Cambridge: Polity Press. 
Brooker, W. (2007). A sort of homecoming: Fan viewing and symbolic pilgrimage. In J. Gray, C. Sandvoss, \& C. L. Harrington (Eds.), Fandom: Identities and communities in a mediated world. London: New York University Press.

Brown, M. E. (1996). Desperately seeking strategies: Reading in the postmodern. In D. Grodin \& R. T. Lindlof (Eds.), Constructing the self in a mediated world (p. 57). Thousand Oaks: Sage Publications.

Cheng, W. (2014). Sound play: Video games and the musical imagination (p. 8). Oxford: Oxford University Press.

D'Errico, M. (2012). How to reformat the planet technostalgia and the 'live' performance of chipmusic. In R. J. Burgess \& K. Isakoff (Eds.), Journal on the art of record production, 6. Conference preceedings from art of production conference 2011. San Francisco, CA: San Francisco State University. http://www.arpjournal.com/asarpwp/how-to-reformat-the-planet-technostalgia-and-the\%E2\%80\%9Clive\%E2\%80\%9D-performance-of-chipmusic/. Accessed July 2018.

Duffett, M. (2013). Understanding fandom: An introduction to the study of media fan culture. London: Bloomsbury Publishing inc.

Frith, S. (2002). Music and identity. In S. Hall \& P. du Gay (Eds.), Questions of cultural identity (p. 110). London: Sage.

Fritsch, M. (2016). 'It's a-me, Mario!'-Playing with video game music. In M. Kamp, T. Summers, \& M. Sweeney (Eds.), Ludomusicology: Approaches to video game music (pp. 92-115). Oxford: Equinox.

Gedalof, I. (1999). Against purity: Rethinking identity with indian and western feminisms (p. 131). London: Routledge

Grossberg, L. (1988). Wandering audiences, nomadic critics. Cultural Studies, 2(3), 337-391.

Grossberg, L. (1992). We gotta get out of this place: Popular conservatism and postmodern culture (p. 82). London: Routledge.

Henry, K., Jusovà, I., \& Westerman, J. (2014). Nomadic encounters: Turning difference towards dialogue. In B. Blaagaard \& I. Van der Tuin (Eds.), The subject of rosi braidotti: Politics and concepts (p. 150). London: Bloomsbury.

Hills, M. (2002). Fan cultures (p. 109). London: Routledge.

Hills, M. (2014). Back in the mix: Exploring intermediary fandom and popular music production. In M. Duffett (Ed.), Popular music fandom: Identities, roles and practices (pp. 18-20). London: Routledge.

Jenkins, H. (2013). textual poachers: Television fans and participatory culture (Twentieth anniversary edition ed., p. 353). London: Routledge.

Latour, B. (2005). Reassembling the social: An introduction to actor-network-theory (p. 45). Oxford: Oxford University Press.

Massumi, B. (2002) Parables for the virtual: Movement, affect, sensation (1. 601, 1089). London: Duke University Press. [Kindle Edition]

Moseley, R. (2016) Keys to play: Music as a ludic medium from apollo to Nintendo (pp. 15-17, 42). California: University of California Press

Nova, N. (2014) 8-Bit reggae: Collision and creolization. Italy: Creative Commons; Near Future Laboratory; Volumique

Peter, B. (2014). Beyond capital, towards myth: EDM fandom and dance practice. In M. Duffett (Ed.), Popular music fandom: Identities, roles and practices (p. 47). London: Routledge.

Sandvoss, C. (2005). Fans: The mirror of consumption (pp. 64-67). Cambridge: Polity Press.

Small, C. (1998) Musicking: The meanings of performing and listening (p. 3, 40). Middletown: Wesleyan University Press

Thornton, S. (1995). Club cultures. Cambridge: Polity Press.

Van Dijck, J. (2007) Mediated memories in the digital age (pp. 1, 27). California: Stanford University Press

Van Elferen, I. (2012) Goth music: The sounds of the uncanny (pp. 27, 30). Cardiff: University of Wales Press

Van Elferen, I. (2016). The ALI model: Towards a theory of game musical immersion. In M. Kamp, T. Summers, \& M. Sweeney (Eds.), Ludomusicology: Approaches to video game music (p. 32). Oxford: Equinox.

Wellman, B. (2004). The glocal village: Internet and community. The Arts and Science Review, 1(1), 26-29.

Wolff, J. (1995). Resident alien: Feminist cultural criticism (p. 119). New Haven: Yale University Press. 